\title{
On Functions of Several Split-Quaternionic Variables
}

\author{
Gueo Grantcharov ${ }^{1}$ and Camilo Montoya ${ }^{2}$ \\ ${ }^{1}$ Department of Mathematics and Statistics, Florida International University, Miami, FL 33199, USA \\ ${ }^{2}$ Department of Mathematics, Indiana University, Bloomington, IN 47405, USA
}

Correspondence should be addressed to Gueo Grantcharov; grantchg@fiu.edu

Received 5 October 2015; Accepted 7 February 2016

Academic Editor: Ricardo Weder

Copyright (C) 2016 G. Grantcharov and C. Montoya. This is an open access article distributed under the Creative Commons Attribution License, which permits unrestricted use, distribution, and reproduction in any medium, provided the original work is properly cited.

Alesker studied a relation between the determinant of a quaternionic Hessian of a function and a specific complex volume form. In this note we show that similar relation holds for functions of several split-quaternionic variables and point to some relations with geometry.

\section{Introduction}

Quaternions are known to have deep relation to the selfdual Yang-Mills equations in mathematical physics. It is also known that the self-duality equations have an "indefinite" version, the basic conformal metric used in their definition has signature $(2,2)$. Such equations are not elliptic, but it is known for more than 30 years that many of the integrable systems arise as a reduction of the indefinite self-duality equations [1]. It is also known that the geometry of a superstring with $N=2$ supersymmetry was shown in $[2,3]$ to be described by a space-time with a pseudo-Kähler metric of signature $(2,2)$, whose curvature satisfies the (anti) selfduality equations.

The split quaternions are indefinite analog of quaternions and play similar role in the indefinite self-duality equations as the quaternions play in the positive definite case. It is well known that spaces with quaternionic-like structures (e.g., quaternionic-kähler and hyperkähler) form an active area of research. One topic in it is developing the notion of quaternionic plurisubharmonic functions [4]. Similar to the quaternionic case, there are geometric structures on manifolds of dimension greater than four, related to the split quaternions. Mathematically, these structures are described by quadruples $(g, I, S, T)$, where $g$ is a signature $(2,2)$ metric and $I, S, T$ are parallel endomorphisms of the tangent bundle with respect to the Levi-Civita connection of $g$, such that

$$
\begin{gathered}
I^{2}=-S^{2}=-1, \\
T=I S=-S I, \\
g(I X, I Y)=-g(S X, S Y)=g(X, Y) .
\end{gathered}
$$

In the literature such structures are called hypersymplectic [5], neutral hyperkähler [6], parahyperkähler [7, 8], pseudohyperkähler [9], and so forth. A more general condition is when $I, S, T$ are parallel with respect to a connection with skew-symmetric torsion; such structures are considered in [10]. One of the features is the existence of a nondegenerate $(2,0)$-form given by $\omega(X, Y)=g(S X, Y)+i g(T X, Y)$. Locally the metric arises from a single function, called potential, similar to the Kähler metrics. The function satisfies $\partial$ 。 $S \circ \bar{\partial} f=\omega$. In the quaternionic case, such potentials in multidimensional quaternionic space $\mathbb{H}^{n}$ correspond to a quaternionic plurisubharmonic functions and were considered from analytical view point first by Alesker [4].

The aim of this paper is to provide an analog of the results in [4] for functions of split-quaternionic variables. Although it is unlikely to find an appropriate definition of plurisubharmonic function because of the indefiniteness, a meaning of determinant of a split-quaternionic-Hermitian matrix can be given. As a main result in the paper we show 
that $\partial \circ S \circ \bar{\partial} f=\operatorname{det}(f) d z_{1} \wedge \cdots \wedge d z_{2 n}$, where det is the Moore determinant of the quaternionic Hessian of $f$. The proof is similar to the proof in [4] and relies on a linear change of variables formula and the density of the delta functions of split-quaternionic hyperplanes in $\mathbb{Z}_{s}^{n}$, which is proven by Graev [11]. We notice also that split-Lagrangian calibrations of [12] can be defined naturally for metrics arising from such functions $f$ for which $\operatorname{det}(f) \neq 0$.

\section{The Split Quaternions and Functions on $\mathbb{M}_{s}^{n}$}

2.1. The Algebra of the Split Quaternions. The split quaternions $\mathbb{H}_{s}$ are spanned over $\mathbb{R}$ by the basis $\{1, i, s, t\}$ with algebraic relations $1^{2}=s^{2}=t^{2}=1, i^{2}=-1$, is $=-s i=t$. The inner product $\langle\cdot, \cdot\rangle$ is defined by $\langle 1,1\rangle=\langle i, i\rangle=1$ and $\langle s, s\rangle=\langle t, t\rangle=-1$ (hence the $(2,2)$ signature). The conjugate of $q=x_{0}+x_{1} i+x_{2} s+x_{3} t$ is $\bar{q}:=x_{0}-x_{1} i-x_{2} s-x_{3} t$, and then $\langle q, q\rangle=q \bar{q}=x_{0}^{2}+x_{1}^{2}-x_{2}^{2}-x_{3}^{2}$.

Another realization of $\mathbb{U}_{s}$ in $\mathbb{H}_{\mathbb{C}}$ given by the following embedding $\tau$ :

$$
\mathbb{\boxplus}_{s} \ni(q=z+w \cdot s) \hookrightarrow \tau(q):=\left(\begin{array}{ll}
\bar{z} & \bar{w} \\
w & z
\end{array}\right) \in \operatorname{End}\left(\mathbb{C}^{2}\right)
$$

whose usefulness becomes obvious when we notice that it preserves the norm; that is,

$$
\|q\|^{2}=\langle q, q\rangle=z \bar{z}-w \bar{w}=\operatorname{det}\left(\begin{array}{cc}
\bar{z} & \bar{w} \\
w & z
\end{array}\right)=\operatorname{det} \tau(q) .
$$
the map

The embedding $\tau$ above is extended to vectors in $\mathbb{H}_{s}^{n}$ by

$$
\tau: \mathbb{T}_{s}^{n} \hookrightarrow \operatorname{End}_{\mathbb{R}}\left(\mathbb{C}^{2 n}\right),
$$

where

$$
\begin{gathered}
q \longmapsto \tau(q):=\left(\tau\left(q_{k}\right)\right)_{k=0, \ldots, n-1}=\left(\begin{array}{c}
\tau\left(q_{0}\right) \\
\vdots \\
\tau\left(q_{k}\right) \\
\vdots \\
\tau\left(q_{n-1}\right)
\end{array}\right), \\
q_{k}:=z_{2 k}+z_{2 k+1} \cdot s, \\
\tau\left(q_{k}\right)=\tau\left(z_{2 k}+z_{2 k+1} \cdot s\right):=\left(\begin{array}{cc}
\bar{z}_{2 k} & \bar{z}_{2 k+1} \\
z_{2 k+1} & z_{2 k}
\end{array}\right)
\end{gathered}
$$

so the embedding looks like

$$
\begin{aligned}
& \mathbb{\mathbb { V } _ { s } ^ { n }} \ni q=\left(\begin{array}{c}
q_{0} \\
\vdots \\
q_{n-1}
\end{array}\right) \longmapsto \tau(q):=\left(\begin{array}{c}
\tau\left(q_{0}\right) \\
\vdots \\
\tau\left(q_{n-1}\right)
\end{array}\right) \\
& =\left(\begin{array}{c}
\left(\begin{array}{cc}
\bar{z}_{0} & \bar{w}_{0} \\
w_{0} & z_{0}
\end{array}\right) \\
\vdots \\
\left(\begin{array}{cc}
\bar{z}_{n-1} & \bar{w}_{n-1} \\
w_{n-1} & z_{n-1}
\end{array}\right)
\end{array}\right) .
\end{aligned}
$$

Similarly for an $n \times n$ split-quaternionic matrix, we define its adjoint matrix as the image of the extension of $\tau$ to $M_{2 n}(\mathbb{C})$ given by

$$
A=\left(A_{i j}\right)_{0 \leq i, j \leq n-1} \longmapsto \tau(A):=\left(\tau\left(A_{i j}\right)\right)_{0 \leq i, j \leq n-1},
$$

where $A_{i j}=a_{i j}+b_{i j} \cdot s$ and $a_{i j}, b_{i j} \in \mathbb{C}$.

A related homomorphism is that of the Study map, which we denote by $\mu$, which can be generalized for a matrix $M_{n}\left(\mathbb{\boxplus}_{s}\right) \ni M=A+B \cdot s$, where $A, B \in M_{n}(\mathbb{C})$ :

$$
M=A+B \cdot s \longmapsto \mu(M):=\left(\begin{array}{ll}
\overline{\tau(A)} & \overline{\tau(B)} \\
\tau(B) & \tau(A)
\end{array}\right) .
$$

The Study map also has an associated determinant, where $\operatorname{det}_{\mathbb{C}}$ is the standard determinant of complex matrices, given by

$$
\operatorname{Sdet}(M):=\operatorname{det}_{\mathbb{C}}(\mu(M)) .
$$

For $q_{k}=z_{2 k}+z_{2 k+1} s$, we have $\tau\left(q_{0} \cdot q_{1}\right)=\tau\left(q_{0}\right) \cdot \tau\left(q_{1}\right)$ and we can check that

$$
\begin{aligned}
\tau\left(q_{0} \cdot q_{1}\right) & =\left(\begin{array}{ll}
\overline{z_{0} z_{1}+w_{0} \bar{w}_{1}} & \overline{z_{0} w_{1}+\bar{z}_{1} w_{0}} \\
z_{0} w_{1}+\bar{z}_{1} w_{0} & z_{0} z_{1}+w_{0} \bar{w}_{1}
\end{array}\right) \\
& =\left(\begin{array}{ll}
\bar{z}_{0} \bar{z}_{1}+\bar{w}_{0} w_{1} & \bar{z}_{0} \bar{w}_{1}+z_{1} \bar{w}_{0} \\
z_{0} w_{1}+\bar{z}_{1} w_{0} & z_{0} z_{1}+w_{0} \bar{w}_{1}
\end{array}\right) \\
& =\left(\begin{array}{ll}
\bar{z}_{0} & \bar{w}_{0} \\
w_{0} & z_{0}
\end{array}\right) \cdot\left(\begin{array}{ll}
\bar{z}_{1} & \bar{w}_{1} \\
w_{1} & z_{1}
\end{array}\right)=\tau\left(q_{0}\right) \cdot \tau\left(q_{1}\right) .
\end{aligned}
$$

So it is natural to expect that this representation extends linearly to matrix groups, that is, the following.

Proposition 1. For any split-quaternionic matrices $A$ and $B$, we have the following:

(1) $\tau(A B)=\tau(A) \tau(B)$ for $A=A^{p \times m}$ and $B=B^{m \times l}$, where the superscripts are the respective dimensions.

(2) For $A \in M_{n}\left(\mathbb{H}_{s}\right), \tau\left(A^{*}\right)=T \tau(A)^{*} T$, where $T:=$ $i \tau(i)=i \operatorname{diag}\left(\begin{array}{cc}-i & 0 \\ 0 & i\end{array}\right)=\operatorname{diag}\left(\begin{array}{cc}1 & 0 \\ 0 & -1\end{array}\right)$. 
(3) For $A \in M_{n}\left(\mathbb{H}_{s}\right), S \tau(A)=\overline{\tau(A)} S$, where $S:=\tau(s)=$ $\operatorname{diag}\left(\begin{array}{ll}0 & 1 \\ 1 & 0\end{array}\right)$. Furthermore, if $A \in U_{n}\left(\mathbb{H}_{s}\right)$ is unitary, then $\tau(A) I \tau(A)^{t}=I$; that is, $A$ is symplectic, and $I:=i \tau(-t)=i \operatorname{diag}\left(\begin{array}{cc}0 & -i \\ i & 0\end{array}\right)=\operatorname{diag}\left(\begin{array}{cc}0 & 1 \\ -1 & 0\end{array}\right)$.

Proof. Let $C=A B$. Then $C_{i j}=\sum_{k=0}^{p-1} A_{i k} B_{k j}$ and by the property above we have

$$
\begin{aligned}
\tau\left(C_{i j}\right) & =\tau\left(\sum_{k=0}^{p-1} A_{i k} B_{k j}\right)=\sum_{k=0}^{p-1} \tau\left(A_{i k} B_{k j}\right) \\
& =\sum_{k=0}^{p-1} \tau\left(A_{i k}\right) \tau\left(B_{k j}\right) \\
& =\left(\tau\left(A_{i 0}\right), \ldots, \tau\left(A_{i(p-1)}\right)\right)\left(\begin{array}{c}
\tau\left(B_{0 j}\right) \\
\vdots \\
\tau\left(B_{(p-1) j}\right)
\end{array}\right) \\
& =\tau\left(\left(A_{i k}\right)_{k=0, \ldots, p-1}\right) \tau\left(\left(B_{k j}\right)_{k=0, \ldots, p-1}\right)^{t} \\
& =\left(\sum_{k=0}^{p-1} \tau\left(A_{i k}\right) \tau\left(B_{k j}\right)\right)_{i j} .
\end{aligned}
$$

Hence

$$
\begin{aligned}
& \tau(C)=\left(\tau\left(C_{i j}\right)\right)=\left(\left(\sum_{k=0}^{p-1} \tau\left(A_{i k}\right) \tau\left(B_{k j}\right)\right)_{i j}\right) \\
& =\tau(A) \cdot \tau(B), \\
& \tau\left(A^{*}\right)=\tau\left(\overline{A^{t}}\right)=\tau\left(\bar{A}_{j i}\right)=\left(\tau\left(\overline{A_{j i}}\right)\right) \\
& =\left(\tau\left(\bar{a}_{j i}^{0}+s \cdot\left(\overline{-a_{j i}^{1}}\right)\right)\right)=\left(\left(\begin{array}{cc}
a_{j i}^{0} & \overline{-a_{j i}^{1}} \\
-a_{j i}^{1} & \overline{a_{j i}^{0}}
\end{array}\right)\right) \\
& =T\left(\left(\begin{array}{cc}
a_{j i}^{0} & \overline{a_{j i}^{1}} \\
a_{j i}^{1} & \overline{a_{j i}^{0}}
\end{array}\right)\right) T=T \overline{\left(\left(\begin{array}{cc}
\overline{a_{i j}^{0}} & \overline{a_{i j}^{1}} \\
a_{i j}^{1} & a_{i j}^{0}
\end{array}\right)\right)^{t}} T \\
& =T \overline{\tau(A)^{t}} T=T \tau(A)^{*} T, \\
& \overline{S \tau(A)}=\operatorname{diag}\left(\begin{array}{ll}
0 & 1 \\
1 & 0
\end{array}\right) \overline{\left(\left(\begin{array}{cc}
\overline{a_{i j}^{0}} & \overline{a_{i j}^{1}} \\
a_{i j}^{1} & a_{i j}^{0}
\end{array}\right)\right)} \\
& =\operatorname{diag}\left(\begin{array}{ll}
0 & 1 \\
1 & 0
\end{array}\right)\left(\left(\begin{array}{ll}
a_{i j}^{0} & a_{i j}^{1} \\
\overline{a_{i j}^{1}} & \frac{a_{i j}^{0}}{a^{0}}
\end{array}\right)\right) \\
& =\left(\left(\begin{array}{cc}
\overline{a_{i j}^{1}} & \overline{a_{i j}^{0}} \\
a_{i j}^{0} & a_{i j}^{1}
\end{array}\right)\right) \\
& =\left(\left(\begin{array}{ll}
\overline{a_{i j}^{0}} & \overline{a_{i j}^{1}} \\
a_{i j}^{1} & a_{i j}^{0}
\end{array}\right)\right) \operatorname{diag}\left(\begin{array}{ll}
0 & 1 \\
1 & 0
\end{array}\right)=\tau(A) S .
\end{aligned}
$$

For the second claim, if the adjoint of $U \in U\left(n, \mathbb{H}_{s}\right)$ is unitary as $(2 n \times 2 n)$ matrix, then we show that $\tau(U)$ is symplectic; that is, $\tau(U) I \tau(U)^{t}=I$

$$
\begin{gathered}
U\left(n, \mathbb{H}_{s}\right) \ni U \Longleftrightarrow U U^{*}=\operatorname{Id}_{n} \Longleftrightarrow \tau\left(U U^{*}\right) \\
=\tau(U) \tau\left(U^{*}\right)=\operatorname{Id}_{2 n},
\end{gathered}
$$

but by (12) we have that $\tau\left(U^{*}\right)=T \tau(U)^{*} T$ and combined with (14):

$$
\begin{aligned}
& (\tau(A) S)^{t}=S \tau(A)^{t}=(\overline{S \tau(A)})^{t}=\overline{\tau(A)^{t}} S \Longrightarrow \\
& S \tau(A)^{t} S=\overline{\tau(A)^{t}}
\end{aligned}
$$

we get that

$$
\begin{aligned}
\operatorname{Id}_{2 n} & =\tau(U) \tau\left(U^{*}\right)=\tau(U) T \overline{\tau(U)^{t}} T \\
& =\tau(U) I S\left[S \tau(U)^{t} S\right] I S=\tau(U) I \tau(U)^{t}(-I)
\end{aligned}
$$

and by multiplying the above equation by $I$ on the right we obtain

$$
\begin{aligned}
I & =\operatorname{Id}_{2 n} I=\left(\tau(U) I \tau(U)^{t}(-I)\right) I \\
& =\tau(U) I \tau(U)^{t}\left(-I^{2}\right)=\tau(U) I \tau(U)^{t}
\end{aligned}
$$

as needed.

Following Wan and Wang [13] we define on $\mathbb{C}^{2 n} \ni$ $z=\left(z_{0}, \ldots, z_{2 n-1}\right)$ the corresponding first-order differential operators that act as partial derivatives with respect to the variable in the $(k \alpha)$ th entry, $k=0, \ldots, 2 n-1$, and $\alpha=0$ or 1 , in (6),

$$
\left(\begin{array}{cc}
\nabla_{00} & \nabla_{01} \\
\nabla_{10} & \nabla_{11} \\
\vdots & \vdots \\
\nabla_{(2 n-2) 0} & \nabla_{(2 n-2) 1} \\
\nabla_{(2 n-1) 0} & \nabla_{(2 n-1) 1}
\end{array}\right):=\left(\begin{array}{cc}
\partial_{\bar{z}_{0}} & \partial_{\bar{z}_{1}} \\
\partial_{z_{1}} & \partial_{z_{0}} \\
\vdots & \vdots \\
\partial_{\bar{z}_{2 n-2}} & \partial_{\bar{z}_{2 n-1}} \\
\partial_{z_{2 n-1}} & \partial_{z_{2 n-2}}
\end{array}\right)
$$

and use them to define the analogs of $\partial$ and $\bar{\partial}$ in complex analysis for the purposes of our study of functions on $\mathbb{T}_{s}^{n}$. We will denote by $\partial_{i}, \partial_{\bar{j}}$ the partial derivatives $\partial_{z_{i}}$ and $\partial_{\bar{z}_{j}}$ for notational simplicity.

Definition 2 (the Baston operator). Let $D \subset \mathbb{R}^{4 n}$ be a domain. The operators $d_{0}, d_{1}$, and $\Delta$ are defined as

$$
\begin{aligned}
d_{0} & :=\left(\omega=\sum_{k} f_{k} d z_{k}\right) \longmapsto d_{0} \omega:=\sum_{k} \nabla_{k 0} \omega d z_{k} \\
d_{1} & :=f \longmapsto d_{1} f:=\sum_{k} \nabla_{k 1} f d z_{k} \\
\Delta & :=d_{0} d_{1}: f \longmapsto \Delta f:=d_{0} d_{1} f .
\end{aligned}
$$


Due to the particular embedding we chose, we can simplify notations and calculations, summarized in the following lemma.

Lemma 3. Properties of $\nabla_{k 0}, \nabla_{k 1}, d_{0}, d_{1}$, and $\Delta$ are as follows:

(1)

$$
\Delta f:=d_{0} d_{1} f=\sum_{0 \leq i<j \leq 2 n-1} \Delta_{i j} f d z_{i} \wedge d z_{j},
$$

where

$$
\Delta_{i j}:=\nabla_{i 0} \nabla_{j 1}-\nabla_{i 1} \nabla_{j 0}=\operatorname{det}\left(\begin{array}{cc}
\nabla_{i 0} & \nabla_{i 1} \\
\nabla_{j 0} & \nabla_{j 1}
\end{array}\right) .
$$

(2) $\Delta_{i j}=-\Delta_{j i}$ for the same pair of indices $i$ and $j$.

(3) Let $I=\{(2 k, 2 k+1) \mid k=0, \ldots, n-1\}=\{(0,1), \ldots$, $(2 n-2,2 n-1)\}$ such that (clearly) $2 k<2 k+1$ for all $k$. Let $r \neq s \in\{0,1, \ldots, n-1\}$; then without loss of generality we can suppose $r<s$. Then if we consider the ordered pairs $\left(i_{0}, i_{1}\right):=(2 r, 2 r+1)$ and $\left(j_{0}, j_{1}\right):=$ $(2 s, 2 s+1)$, we have

$$
\Delta_{i_{0} j_{1}}=-\overline{\Delta_{i_{1} j_{0}}} .
$$

Proof. We compute $\Delta f$ as

$$
\begin{aligned}
\Delta f & :=d_{0}\left(d_{1} f\right)=d_{0}\left(\sum_{j=0}^{2 n-1} \nabla_{j 1} f d z_{j}\right) \\
& =\left(\sum_{i=0}^{2 n-1} \nabla_{i 0} d z_{i}\right) \wedge\left(\sum_{j=0}^{2 n-1} \nabla_{j 1} f d z_{j}\right) \\
& =\sum_{i, j=0}^{2 n-1} \nabla_{i 0} \nabla_{j 1} f d z_{i} \wedge d z_{j} .
\end{aligned}
$$

Since both indices $i$ and $j$ run from 0 to $2 n-1$, the "symmetric terms" $\nabla_{i 0} \nabla_{j 1} f d z_{i} \wedge d z_{j}$ and $\nabla_{j 0} \nabla_{i 1} f d z_{j} \wedge d z_{i}$ both appear, and by the skew-symmetry of the wedge product $\left(d z_{i} \wedge d z_{j}=-d z_{j} \wedge d z_{i}\right)$,

$$
\begin{aligned}
\nabla_{i 0} & \nabla_{j 1} f d z_{i} \wedge d z_{j}+\nabla_{i 1} \nabla_{j 0} f d z_{j} \wedge d z_{i} \\
& =\left(\nabla_{i 0} \nabla_{j 1} f-\nabla_{i 1} \nabla_{j 0} f\right) d z_{i} \wedge d z_{j} \Longrightarrow \\
\Delta f & =\sum_{i, j=0}^{2 n-1} \nabla_{i 0} \nabla_{j 1} f d z_{i} \wedge d z_{j} \\
& =\frac{1}{2} \sum_{i, j=0}^{2 n-1}\left(\nabla_{i 0} \nabla_{j 1} f-\nabla_{i 1} \nabla_{j 0} f\right) f d z_{i} \wedge d z_{j}
\end{aligned}
$$

and since any $d z_{k} \wedge d z_{k}=0$, the only remaining terms are the terms with $0 \leq i<j \leq 2 n-1$, so we can cancel the $1 / 2$ and write (19) as

$$
\begin{aligned}
\Delta f & =\sum_{0 \leq i<j \leq 2 n-1}\left(\nabla_{i 0} \nabla_{j 1} f-\nabla_{i 1} \nabla_{j 0} f\right) d z_{i} \wedge d z_{j} \\
& =\sum_{0 \leq i<j \leq 2 n-1} \Delta_{i j} f d z_{i} \wedge d z_{j},
\end{aligned}
$$

where $\Delta_{i j}$ is the coefficient of $d z_{i} \wedge d z_{j}$ th term in $\Delta f$.
To demonstrate (2), we use commutation of $\nabla_{k \alpha} \nabla_{j \beta}=$ $\nabla_{j \beta} \nabla_{k \alpha}$ :

$$
\begin{aligned}
-\Delta_{j i} & :=-\left(\nabla_{j 0} \nabla_{i 1}-\nabla_{j 1} \nabla_{i 0}\right)=\nabla_{j 1} \nabla_{i 0}-\nabla_{j 0} \nabla_{i 1} \\
& =\nabla_{i 0} \nabla_{j 1}-\nabla_{i 1} \nabla_{j 0}=\Delta_{i j} .
\end{aligned}
$$

Proving the final claim is done in two nearly identical calculations, depending on the parity of $r$ and $s$. We will prove one case, where without loss of generality we assume $r$ is even and we still have $r<s$. This means that $\nabla_{i_{0} 0}=\nabla_{(2 r) 0}=\partial_{\overline{2 r}}$, $\nabla_{i_{1} 0}=\nabla_{(2 r+1) 0}=\partial_{2 r+1}$, but $\nabla_{i_{0} 1}=\nabla_{(2 r) 1}=\partial_{2 r+1}, \nabla_{i_{1} 1}=$ $\nabla_{(2 r+1) 1}=\partial_{2 r}$, and similarly $\nabla_{j_{0} 0}=\nabla_{(2 s) 0}=\partial_{\overline{2 s}}, \nabla_{j_{1} 0}=$ $\nabla_{(2 s+1) 0}=\partial_{2 s+1}$, but $\nabla_{j_{0} 1}=\nabla_{(2 s) 1}=\partial_{2 s+1}, \nabla_{j_{1} 1}=\nabla_{(2 s+1) 1}=\partial_{2 s}$ (in the other case if $s$ was odd we would have the completely symmetric situation, with $2 r+1$ 's becoming $\overline{2 r}$ 's, etc.). So we calculate

$$
\begin{aligned}
-\overline{\Delta_{i_{1} j_{0}}} & =\overline{-\Delta_{i_{1} j_{0}}}=\overline{\Delta_{j_{0} i_{1}}}=\overline{\nabla_{j_{0} 0} \nabla_{i_{1} 1}-\nabla_{j_{0} 1} \nabla_{i_{1} 0}} \\
& =\overline{\partial_{\overline{2 s}} \partial_{2 r}-\partial_{\overline{2 s+1}} \partial_{2 r+1}}=\overline{\partial_{\overline{2 s}} \partial_{2 r}}-\overline{\partial_{\overline{2 s+1}} \partial_{2 r+1}} \\
& =\partial_{\overline{2 r}} \partial_{2 s}-\partial_{\overline{2 r+1}} \partial_{2 s+1} \\
& =\nabla_{(2 r) 0} \nabla_{(2 s+1) 1}-\nabla_{(2 r) 1} \nabla_{(2 s+1) 0}=: \Delta_{(2 r)(2 s+1)} \\
& =\Delta_{i_{0} j_{1}} .
\end{aligned}
$$

Definition 4 (mixed Baston product). For $f_{1}, \ldots, f_{n} \in C^{2}$ we define the mixed Baston product of $f_{1}, \ldots, f_{n}$ as

$$
\begin{aligned}
\Delta_{n} & \left(f_{1}, \ldots, f_{n}\right):=\Delta f_{1} \wedge \Delta f_{2} \wedge \cdots \wedge \Delta f_{n} \\
:= & \sum_{i_{1}, j_{1}, \ldots} \Delta_{i_{1} j_{1}} f_{1} \cdots \Delta_{i_{n} j_{n}} f_{n} d z_{i_{1}} \wedge d z_{j_{1}} \wedge \cdots \wedge d z_{i_{n}} \\
& \wedge d z_{j_{n}} \\
= & \sum_{i_{1}, j_{1}, \ldots} \delta_{1,2,3,4, \ldots, 2 n}^{i_{1} j_{1} i_{2}, \ldots, i_{n} j_{n}} \Delta_{i_{1} j_{1}} f_{1} \cdots \Delta_{i_{n} j_{n}} f_{n} d z_{I},
\end{aligned}
$$

where $I=\{1,2, \ldots, 2 n\}$ and $\delta_{1,2,3,4, \ldots, 2 n}^{i_{1} j_{1} i_{2} j_{2}, \ldots, i_{n} j_{n}}$ is defined to be the sign of the permutation from $\left(i_{1}, j_{1}, \ldots, i_{n}, j_{n}\right)$ to $(1,2,3, \ldots, 2 n)$ if $\left\{i_{k}, j_{k} \mid k=1,2, \ldots, 2 n\right\}=I$ and 0 otherwise.

In particular, for $f_{1}=f_{2}=\cdots=f_{n}=f$, the mixed Baston product coincides with the $n$-times wedged Baston of $f$; that is,

$$
\begin{aligned}
\Delta_{n}(f) & :=\Delta_{n}(f, \ldots, f)=(\Delta f)^{n}:=: \bigwedge^{n} \Delta f \\
& :=\underbrace{(\Delta f) \wedge \cdots \wedge(\Delta f) .}_{n \text {-times }}
\end{aligned}
$$

The results and definitions above will allow us to translate the $n$-times wedged Baston of $f$ in terms of the "splitquaternionic Hessian" of $f$, defined in terms of the Moore determinant, which are precisely the next sections. 
2.2. Split-Quaternionic Determinants. Due to the noncommutativity of the multiplication in $\mathbb{U}_{s}$ (just as in $\mathbb{H}$ ) trying to construct an effective definition of determinants is complicated. There are several ways to define them. The main results in this direction follow the work of E. Study, J. Dieudonné, and E. H. Moore, as outlined by Aslaksen in [14]. However, the problem becomes much simpler if we are restricted to hyperhermitian matrices; that is, $A \in$ $\mathrm{GL}_{n}\left(\mathbb{H}_{s}\right)$ such that $A=A^{*}$; then we can define a simple and useful determinant following the work of E. H. Moore, called the Moore determinant. This is done by specifying a certain ordering of the factors in $n$ ! terms in the sum over permutations of the symmetric group $S_{n}$.

Definition 5 (the Moore determinant, see [14] or [15]). For a permutation $\sigma \in S_{n}$, write $\sigma$ as a product of disjoint cycles such that the smallest number is at the front of each factor and then sort the disjoint cycles in decreasing order according to the first number of each factor. In other words, write

$$
\sigma=\left(n_{11} \cdots n_{1 r_{1}}\right)\left(n_{21} \cdots n_{2 r_{2}}\right) \cdots\left(n_{j 1} \cdots n_{j r_{j}}\right),
$$

where, for $i$, we have $n_{i 1}>n_{i k}$ for all $k>1$, and $n_{11}>$ $n_{21}>\cdots>n_{j 1}$. Then we define the Moore determinant of a hyperhermitian matrix $A=\left(a_{i j}\right)$ [denoted by $\left.\operatorname{det}(A)\right]$ as

$$
\operatorname{det}(A):=\sum_{\sigma \in S_{n}}|\sigma| a_{n_{11} n_{12}} \cdots a_{n_{1 r_{1}} n_{11}} a_{n_{21} n_{22}} \cdots a_{n_{j r_{j}} n_{j 1}} .
$$

Another equivalent definition of the Moore determinant is the inductive one (see [15]), defined as, for a hyperhermitian $n \times n$ matrix $A=\left(a_{i j}\right)$, the inductive definition is given as follows: for $n=1$, we have $\operatorname{det}(A):=a_{11}$ and for $n>2$

$$
\operatorname{det}(A)=\sum_{k=1}^{n} \epsilon_{r k} a_{r k} \operatorname{det}(A(r, k))
$$

for $r \in I=\{1,2, \ldots, n\}, \epsilon_{r k}=+1$ if $k=r$ and $\epsilon_{r k}=-1$ if $k \neq r$, and $A(k, r)$ the hyperhermitian $(n-1) \times(n-1)$ matrix obtained by interchanging the $k$ th and $r$ th columns and then deleting both the $k$ th row and column of the corresponding matrix. For any matrix $A \in M_{n}\left(\mathbb{U}_{s}\right)$, it can be easily checked that $A \cdot A^{*}=A^{*} \cdot A$ is also hyperhermitian, which leads to the equalities

$$
\operatorname{det}\left(A^{*} \cdot A\right)=\operatorname{det}_{\mathbb{C}}(\mu(A)) .
$$

The Moore determinant is related to the Study determinant from (8) as

$$
\begin{aligned}
\operatorname{det}\left(A^{*} \cdot A\right) & =\operatorname{det}_{\mathbb{C}}(\tau(A))=\operatorname{det}_{\mathbb{C}}(\mu(A)) \\
& =\operatorname{Sdet}(A),
\end{aligned}
$$

which is given by the middle equality which can be seen easily by noticing that $\mu(A)$ and $\tau(A)$ are similar matrices (having the same exact entries in different arrays, except that the former consists of $4 n$-blocks and the latter consists of $n^{2} 2$-blocks) and differ by only elementary operations (shuffling some rows, columns, and signs) so that their complex determinants are equal.
Again focusing on hyperhermitian matrices, we can manipulate them to get what are also known as self-adjoint matrices, for which the Pfaffians (Pf) can be defined (again see [15]). They are defined on $2 n \times 2 n$ skew-symmetric matrices, so for a hyperhermitian matrix $A$ and matrix (and endomorphism) $T$ defined in Proposition 1, we define the map $\rho$ by

$$
A \longmapsto \rho(A):=T \cdot \tau(A) .
$$

From this follows the well-known equalities proved by Dyson [15]:

$$
\begin{aligned}
\operatorname{det}(A)=\operatorname{Pf}(\rho(A))= & \sqrt{\operatorname{Sdet}(A)} \\
& \text { i.e., }[\operatorname{det}(A)]^{2}=\operatorname{det}_{\mathbb{C}}(\tau(A)) .
\end{aligned}
$$

This allows us to prove det is a homomorphism and, in particular, the following corollary.

Corollary 6. For any hyperhermitian matrix $A$ and any split-quaternionic matrix $C$, the matrix $C^{*} \cdot A \cdot C$ is also hyperhermitian and

$$
\operatorname{det}\left(C^{*} \cdot A \cdot C\right)=\operatorname{det}\left(C^{*} \cdot C\right) \cdot \operatorname{det}(A) .
$$

Proof. Using the identities (32), (33), and (35) above, Proposition 1, and the multiplicative properties of complex determinants and $\tau$, a direct calculation shows

$$
\begin{aligned}
& {\left[\operatorname{det}\left(C^{*} \cdot A \cdot C\right)\right]^{2}=\operatorname{det}_{\mathbb{C}}\left(\tau\left(C^{*} \cdot A \cdot C\right)\right)} \\
& \quad=\operatorname{det}_{\mathbb{C}}\left(\tau\left(\left(C^{*}\right) \cdot \tau(A) \cdot \tau(C)\right)\right) \\
& =\operatorname{det}_{\mathbb{C}}\left(\tau\left(C^{*}\right)\right) \cdot \operatorname{det}_{\mathbb{C}}(\tau(A)) \cdot \operatorname{det}_{\mathbb{C}}(\tau(C)) \\
& =\operatorname{det}_{\mathbb{C}}\left(\tau\left(C^{*}\right)\right) \operatorname{det}_{\mathbb{C}}(\tau(C)) \operatorname{det}_{\mathbb{C}}(\tau(A)) \\
& =\left[\operatorname{det}_{\mathbb{C}}\left(\tau\left(C^{*} \cdot C\right)\right)\right] \cdot \operatorname{det}_{\mathbb{C}}(\tau(A)) \\
& =\left[\operatorname{det}_{(}\left(C^{*} \cdot C\right)\right]^{2} \cdot[\operatorname{det}(A)]^{2}
\end{aligned}
$$

and the corollary is proved.

Definition 7 (mixed discriminant of hyperhermitian matrices). Let $A_{1}, \ldots, A_{n}$ be hyperhermitian $n \times n$ matrices. The mixed discriminant [denoted by $\operatorname{det}\left(A_{1}, \ldots, A_{n}\right)$ ] of $A_{1}, \ldots, A_{n}$ is defined to be the coefficient of the monomial $x_{1}, \ldots, x_{n}$ divided by $n$ ! in the polynomial given by $\operatorname{det}\left(x_{1} A_{1}+\right.$ $\left.\cdots+x_{n} A_{n}\right)$, where det is again the Moore determinant. Note also that $\operatorname{det}(A, \ldots, A)=\operatorname{det}(A)$.

2.3. The "Split-Quaternionic Hessian" of a Function. Let $f$ be a function of $n$ split-quaternionic variables, where $q_{i}:=z_{2 i}+$ $\bar{z}_{2 i+1} \cdot s$. We define the partial derivative with respect to $q_{i}$ as

$$
\frac{\partial}{\partial q_{i}}:=\partial_{2 i}+\partial_{\overline{2 i+1}} \cdot s
$$

and for $q_{j}:=z_{2 j}+z_{2 j+1} \cdot s$, its conjugate is $\bar{q}_{j}:=\bar{z}_{2 j}-\bar{z}_{2 j+1}$. $s$, we similarly define the partial derivative with respect to a conjugate variable as

$$
\frac{\partial}{\partial \bar{q}_{j}}:=\frac{\bar{\partial}}{\partial q_{j}}:=\partial_{\overline{2 j}}-\partial_{\overline{2 j+1}} \cdot s .
$$


This immediately implies that the mixed partials calculation is

$$
\begin{aligned}
\frac{\partial^{2}}{\partial q_{i} \partial \bar{q}_{j}}:= & \frac{\partial}{\partial q_{i}}\left(\frac{\partial}{\partial q_{j}}\right) \\
:= & \left(\partial_{2 i}+\partial_{\overline{2 i+1}} \cdot s\right)\left(\partial_{\overline{2 j}}-\partial_{\overline{2 j+1}} \cdot s\right) \\
= & \partial_{2 i} \partial_{\overline{2 j}}-\partial_{2 i} \partial_{\overline{2 j+1}} \cdot s+\partial_{\overline{2 i+1}} \cdot s \cdot \partial_{\overline{2 j}}-\partial_{\overline{2 i+1}} \cdot s \\
& \cdot \partial_{\overline{2 j+1}} \cdot s \\
= & \partial_{2 i} \partial_{\overline{2 j}}-\partial_{\overline{2 i+1}} \partial_{2 j+1}+\left(\partial_{\overline{2 i+1}} \partial_{2 j}-\partial_{2 i} \partial_{\overline{2 j+1}}\right) \cdot s \\
= & \partial_{(2 i)(\overline{2 j})}^{2}-\partial_{\overline{(2 i+1)}(2 j+1)}^{2} \\
& +\left(\partial_{\overline{(2 i+1)}(2 j)}^{2}-\partial_{(2 i) \overline{(2 j+1)}}^{2}\right) \cdot s
\end{aligned}
$$

since $s \cdot \partial_{k}=\partial_{\bar{k}} \cdot s$ which extends from the fact that for $z \in \mathbb{C}$ and $s \in \mathbb{H}_{s}, s \cdot z=\bar{z} \cdot s$. This also shows that the matrix $\left(\partial^{2} / \partial q_{i} \partial \bar{q}_{j}\right)_{i, j=0, \ldots, n-1}$ is hyperhermitian; that is,

$$
\frac{\partial^{2}}{\partial q_{i} \partial \bar{q}_{j}}=\overline{\frac{\partial^{2}}{\partial q_{j} \partial \bar{q}_{i}}}
$$

Definition 8 (the "split-quaternionic Hessian" of a function $f)$. The "split-quaternionic Hessian" (denoted $\mathscr{H}_{s}$ ) of a $C^{2}$ function defined on a domain $D$ in $\mathbb{H}_{s}^{n} \cong \mathbb{C}^{2 n}$ is defined analogously to the complex Hessian of a function, only with respect to split-quaternionic variables. For $i, j \in\{0,1, \ldots, n-$ $1\}$

$$
\begin{aligned}
\partial_{i j}^{q}:=\frac{\partial^{2}}{\partial q_{i} \partial \bar{q}_{j}}: & \\
f & \longmapsto \partial_{i j}^{q}(f):=\frac{\partial^{2}}{\partial q_{i} \partial \bar{q}_{j}}(f):=\frac{\partial^{2} f}{\partial q_{i} \partial \bar{q}_{j}},
\end{aligned}
$$

and $\mathscr{H}_{s}(f)$, the split-quaternionic Hessian of $f$, is defined as

$$
\begin{aligned}
\mathscr{H}_{s}: C^{2}(D) & \longrightarrow M_{n}\left(\mathbb{H}_{s}\right), \\
f & \longmapsto \mathscr{H}_{s}(f):=\left[\partial_{i j}^{q}(f)\right]_{i, j=0,1, \ldots, 2 n-1} .
\end{aligned}
$$

We now turn to the Monge-Amperè operator. As Alesker in [4] defined the mixed Monge-Amperè operator in quaternionic space of a $C^{2}$ function $f$ is defined as

$$
\operatorname{MA}(f): f \longmapsto \operatorname{det}\left(\mathscr{H}_{s}(f)\right) \text {. }
$$

Generalized further, for $C^{2}$ functions $f_{1}, \ldots, f_{n}$, the mixed discriminant $\mathrm{ds}$ is defined as the Moore determinant of the respective quaternionic Hessian matrices; we follow this construction to define a similar Monge-A-Amperè operator to split-quaternionic functions, denoted by

$$
\begin{aligned}
& \operatorname{MA}\left(f_{1}, \ldots, f_{n}\right):=\operatorname{det}\left(f_{1}, \ldots, f_{n}\right) \\
& :=\operatorname{det}\left(\left(\frac{\partial^{2} f_{1}}{\partial q_{i} \partial \bar{q}_{j}}\right), \ldots,\left(\frac{\partial^{2} f_{n}}{\partial q_{i} \partial \bar{q}_{j}}\right)\right) .
\end{aligned}
$$

Note also that, for $f_{1}=f_{2}=\cdots=f_{n}=f$, the mixed Monge-Amperè operator is equal to the regular MongeAmperè operator:

$$
\operatorname{det}(f):=\operatorname{det}(f, \ldots, f)=\operatorname{det} \mathscr{H}_{s}(f) .
$$

Lemma 9. For any hyperhermitian $n \times n$ matrix $A$ and any real diagonal real matrix of the form $T=\left(\begin{array}{ccc}t_{1} & & 0 \\ & \ddots & \\ 0 & & t_{n}\end{array}\right)$ we have that

$$
\operatorname{det}(A+T)=\sum_{I \subset\{1, \ldots, n\}}\left(\prod_{i \in I} t_{i}\right) \cdot \operatorname{det}\left(M_{I} A\right),
$$

where $M_{I} A$ is the matrix obtained by deleting rows and columns with indexes from a nonempty subset $I \subset\{1,2, \ldots, n\}$ (see [4], pg. 10).

This lemma is proved as proposition 1.1.11 in Alesker [4]; we will just be using a simple corollary.

Corollary 10. If $t_{2}=\cdots=t_{n}=0$ in Lemma 9 above, then

$$
\operatorname{det}(A+T)=\operatorname{det}(A)+t_{1} \cdot \operatorname{det}\left(M_{\{1\}} A\right)
$$

where

$$
A=\left(\begin{array}{cc}
a_{11} & * \\
* & M_{\{1\}} A
\end{array}\right)
$$

and $M_{\{1\}} A$ is still a hyperhermitian $(n-1) \times(n-1)$ matrix, $a_{11} \in \mathbb{R}$.

2.4. Linear Change of Variables. In this section we prove a split-quaternionic change of variables formula for linear transformations. Since the split quaternions can be represented by real $(2 \times 2)$ matrices, this endeavour is done easier via a real representation of the matrix algebra. In this light we define $\tau_{\mathbb{R}}$ to be the following embedding (also a homomorphism like $\tau$, see Proposition 1) for a splitquaternionic vector $q=\left(q_{k}\right)=\left(x_{4 k}+x_{4 k+1} i+x_{4 k+2} s+x_{4 k+3} t\right) \epsilon$ $\mathbb{H}_{s}^{n}$ and matrix $A=A_{0}+A_{1} i+A_{2} s+A_{3} t$, where $A_{i} \in M_{n}(\mathbb{R})$ :

$$
\begin{aligned}
\mathbb{H}_{s}^{n} \ni & q \longmapsto \tau_{\mathbb{R}}(q) \\
\mathrm{GL}_{n}\left(\mathbb{H}_{s}\right) & \left.\ni A \longmapsto \tau_{4 k}, x_{4 k+1}, x_{4 k+2}, x_{4 k+3}\right)_{k=0,1, \ldots, n-1}^{t} \in \mathbb{R}^{4 n}, \\
& :=\left(\begin{array}{cccc}
A_{0} & -A_{1} & A_{2} & A_{3} \\
A_{1} & A_{0} & A_{3} & -A_{2} \\
A_{2} & A_{3} & A_{0} & -A_{1} \\
A_{3} & -A_{2} & A_{1} & A_{0}
\end{array}\right) \in M_{4 n}(\mathbb{R})
\end{aligned}
$$


Using this real embedding the corresponding matrices $I_{\mathbb{R}}, S_{\mathbb{R}}$, and $T_{\mathbb{R}}$ are

$$
\begin{aligned}
I_{\mathbb{R}}:= & \left(\begin{array}{cccc}
0 & -1 & 0 & 0 \\
1 & 0 & 0 & 0 \\
0 & 0 & 0 & 1 \\
0 & 0 & -1 & 0
\end{array}\right), \\
S_{\mathbb{R}}:= & \left(\begin{array}{cccc}
0 & 0 & 1 & 0 \\
0 & 0 & 0 & 1 \\
1 & 0 & 0 & 0 \\
0 & 1 & 0 & 0
\end{array}\right), \\
T_{\mathbb{R}}:= & \left(\begin{array}{cccc}
0 & 0 & 0 & 1 \\
0 & 0 & -1 & 0 \\
0 & -1 & 0 & 0 \\
1 & 0 & 0 & 0
\end{array}\right)
\end{aligned}
$$

and satisfy the split-quaternionic relations (1). Moreover, if we define

$$
\mathbb{q}_{\alpha}:=\operatorname{diag}\left(I_{\alpha}\right), \quad \alpha=1,2,3
$$

for $I_{1}=I_{\mathbb{R}}, I_{2}=S_{\mathbb{R}}, I_{3}=T_{\mathbb{R}}$, then one can calculate that $\tau_{\mathbb{R}}(A)$ commutes with $\rrbracket_{\alpha}$; that is

$$
\rrbracket_{\alpha} \cdot \tau_{\mathbb{R}}(A)=\tau_{\mathbb{R}}(A) \cdot \rrbracket_{\alpha}, \quad \alpha=1,2,3 .
$$

For a $C^{1}$ function $f: \mathbb{H}_{s}^{n} \rightarrow \mathbb{H}_{s}, f=f_{0}+f_{1} i+f_{2} s+f_{3} t$, and the real representation denoted by $f^{\mathbb{R}}=\left(f_{0}, f_{1}, f_{2}, f_{3}\right)^{t}$, the partial derivative of $f$ with respect to $q_{j}$ can be written as the $(4 \times 4)$ differential operator

$$
\begin{aligned}
\left(\partial_{q_{j}} f\right)^{\mathbb{R}} & :=\left(\frac{\partial f}{\partial q_{j}}\right)^{\mathbb{R}} \\
& :=\left(\begin{array}{cccc}
\partial_{x_{4 j}} & -\partial_{x_{4 j+1}} & \partial_{x_{4 j+2}} & \partial_{x_{4 j+3}} \\
\partial_{x_{4 j+1}} & \partial_{x_{4 j}} & \partial_{x_{4 j+3}} & -\partial_{x_{4 j+2}} \\
\partial_{x_{4 j+2}} & \partial_{x_{4 j+3}} & \partial_{x_{4 j}} & -\partial_{x_{4 j+1}} \\
\partial_{x_{4 j+3}} & -\partial_{x_{4 j+2}} & \partial_{x_{4 j+1}} & \partial_{x_{4 j}}
\end{array}\right) f^{\mathbb{R}}
\end{aligned}
$$

and the derivative in the direction $q \in \mathbb{H}_{s}^{n}$ as

$$
\partial_{q} f:=\mathscr{D}_{x} f^{\mathbb{R}}:=\left(\begin{array}{c}
\left(\partial_{q_{0}} f\right)^{\mathbb{R}} \\
\vdots \\
\left(\partial_{q_{n-1}} f\right)^{\mathbb{R}}
\end{array}\right)
$$

For a linear transformation $A \in \mathrm{GL}_{n}\left(\mathbb{H}_{s}\right) ; A=\left(a_{i j}\right): \mathbb{H}_{s}^{n} \rightarrow$ $\mathbb{H}_{s}^{n} ; q \mapsto q^{\prime}:=A q$ and a $C^{1}$ function $f^{\prime}: \mathbb{T}_{s}^{n} \rightarrow \mathbb{H}_{s}$ we define the pullback via $A$ of $f^{\prime}$ as $f(q):=f^{\prime}(A q)\left[=f^{\prime}\left(q^{\prime}\right)\right]$ and their corresponding real representation denoted as $f^{\prime} \mathbb{R}$ and
$f^{\mathbb{R}}$. Let $x=\tau_{\mathbb{R}}(q)$ and $y=\tau_{\mathbb{R}}\left(q^{\prime}\right)$, so that $y=\tau_{\mathbb{R}}(A) x$, and $f(q)=f^{\prime}(A q)$, that is, $f^{\mathbb{R}}(x)=f^{\prime} \mathbb{R}\left(\tau_{\mathbb{R}}(A) x\right)$

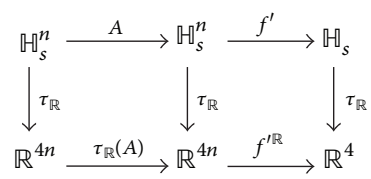

Proposition 11. With the same setup as above, we have

$$
\mathscr{D}_{x} f^{\mathbb{R}}(x)=\left(\tau_{\mathbb{R}}\left(A^{*}\right)\right) \mathscr{D}_{y} f^{\prime} \mathbb{R}\left(\tau_{\mathbb{R}}(A) x\right) ;
$$

that is,

$$
\partial_{q_{j}} f(q)=\sum_{k=0}^{n-1} \bar{a}_{k j} \partial_{q_{k}^{\prime}} f^{\prime}(A q)
$$

Proof. Denote by $\left(\partial_{q_{j}}\right)^{\alpha}$ the $\alpha$ th column of the functional operator $\partial_{q_{j}}: f \mapsto\left(\partial_{q_{j}} f\right)^{\mathbb{R}}$, for $\alpha=1,2,3,4$, and then by the definitions of $\left(\partial_{q_{j}}\right)^{\alpha}$ and $I_{\alpha}$ it follows directly that

$$
\left(\partial_{q_{j}}\right)^{\alpha}=I_{\alpha-1}\left(\begin{array}{l}
\partial_{x_{4 j}} \\
\partial_{x_{4 j+1}} \\
\partial_{x_{4 j+2}} \\
\partial_{x_{4 j+3}}
\end{array}\right), \quad \alpha=1,2,3,4
$$

with the understanding that $I_{0}=\operatorname{Id}_{4 \times 4}$ for the $\alpha=1$ th column. Hence we have

$$
\left(\mathscr{D}_{x}\right)^{\alpha}=\mathbb{\square}_{\alpha-1} \partial_{x}=\rrbracket_{\alpha-1}\left(\left(\begin{array}{c}
\partial_{x_{4 j}} \\
\partial_{x_{4 j+1}} \\
\partial_{x_{4 j+2}} \\
\partial_{x_{4 j+3}}
\end{array}\right)\right)_{j=0, \ldots, n-1}
$$

where $\left(\mathscr{D}_{x}\right)^{\alpha}$ is the $\alpha$ th column of the functional operator $\mathscr{D}_{x}: f \mapsto\left(\mathscr{D}_{x} f\right)^{\mathbb{R}}$. Since $y=\tau_{\mathbb{R}}(A) x$, then by definition we have $y_{k}=\sum_{m=0}^{4 n-1}\left(\tau_{\mathbb{R}}(A)\right)_{k m} x_{m}$ so that by the chain rule for functions of several variables

$$
\begin{aligned}
\partial_{x_{j}} f^{\mathbb{R}}(x) & =\sum_{k=0}^{4 n-1}\left(\tau_{\mathbb{R}}(A)\right)_{k j} \partial_{y_{k}} f^{\prime} \mathbb{R}\left(\left(\tau_{\mathbb{R}}(A)\right) x\right) \Longrightarrow \\
\left(\mathscr{D}_{x} f\right)^{\mathbb{R}} & =\left[\tau_{\mathbb{R}}(A)\right]^{t} \cdot \partial_{y} f^{\prime} \mathbb{R}\left(\tau_{\mathbb{R}}(A) x\right)
\end{aligned}
$$

directly in the first column, that is, $\left(\mathscr{D}_{x} f\right)^{1}(x)=$ $\left[\tau_{\mathbb{R}}(A)\right]^{t}\left(\mathscr{D}_{y} f^{\prime}\right)^{1}\left(\tau_{\mathbb{R}}(A) x\right)$. Since $f=f_{0}+f_{1} i+f_{2} s+f_{3} t$ and 
$\mathscr{D}_{x}$ is a linear operator, we use the commutation relations (53) to calculate

$$
\begin{aligned}
\left(\mathscr{D}_{x} f\right)^{\alpha}(x) & =\mathbb{q}_{\alpha-1} \partial_{x} f^{\mathbb{R}}(x) \\
& =\mathbb{q}_{\alpha-1}\left(\left[\tau_{\mathbb{R}}(A)\right]^{t} \partial_{y} f^{\prime \mathbb{R}}\left(\tau_{\mathbb{R}}(A) x\right)\right) \\
& =\left[\tau_{\mathbb{R}}(A)\right]^{t}\left(\mathbb{q}_{\alpha-1} \partial_{y} f^{\prime} \mathbb{R}\left(\tau_{\mathbb{R}}(A) x\right)\right) \\
& =\left[\tau_{\mathbb{R}}(A)\right]^{t}\left(\left(\mathscr{D}_{y} f^{\prime}\right)^{\alpha}\left(\tau_{\mathbb{R}}(A) x\right)\right) \\
& =\left[\tau_{\mathbb{R}}(A)\right]^{t}\left(\mathscr{D}_{y} f^{\prime}\right)^{\alpha}(y),
\end{aligned}
$$

for $\alpha=2,3,4$.

And hence

$$
\begin{aligned}
\left(A^{*} \partial_{q^{\prime}} f^{\prime}\right)^{\mathbb{R}}(A q) & =\tau_{\mathbb{R}}(A)^{t} \mathscr{D}_{y} f^{\prime \mathbb{R}}(y) \\
& =\tau_{\mathbb{R}}(A)^{t} \mathscr{D}_{y} f^{\mathbb{R}}\left(\tau_{\mathbb{R}}(A) x\right) \\
& =\left(\mathscr{D}_{x} f^{\mathbb{R}}\right)(x)=\left(\partial_{q} f\right)^{\mathbb{R}}(q)
\end{aligned}
$$

and (57) follows.

Corollary 12 (change of variables under split-quaternionic linear transformations). If $f$ is a real-valued $C^{2}$ function, then

$$
\left(\frac{\partial^{2} f}{\partial q_{j} \partial \bar{q}_{k}}(q)\right)=A^{*}\left(\frac{\partial^{2} f^{\prime}}{\partial q_{j}^{\prime} \partial \bar{q}_{k}^{\prime}}(A q)\right) A .
$$

Proof. If $u$ is real-valued, then $\partial_{\bar{q}_{k}} u=\overline{\partial_{q_{k}} u}$. Then by (58)

$$
\partial_{q_{i}} u_{j}(q)=\sum_{k=0}^{n-1} \bar{a}_{k i} \partial_{q_{k}^{\prime}} u_{j}^{\prime}(A q)
$$

and taking the conjugate of both sides gives us

$$
\begin{aligned}
\partial_{\bar{q}_{i}} u_{j}(q) & =\overline{\partial_{q_{i}} u_{j}(q)}=\overline{\sum_{k=0}^{n-1} \bar{a}_{k i} \partial_{q_{k}^{\prime}} u_{j}^{\prime}(A q)} \\
& =\sum_{k=0}^{n-1} \overline{\partial_{q_{k}^{\prime}} u_{j}^{\prime}(A q)} a_{k i}=\sum_{k=0}^{n-1} \partial_{\bar{q}_{k}^{\prime}} u_{j}^{\prime}(A q) a_{k i} .
\end{aligned}
$$

Then applying (58) to the LHS of the line above

$$
\begin{array}{r}
\sum_{j, k=0}^{n-1} \bar{a}_{j i} \frac{\partial^{2} u}{\partial q_{j} \partial \bar{q}_{k}}(A q) a_{k i}=\sum_{k=0}^{n-1} \frac{\partial f_{k}}{\partial q_{j}^{\prime}}(q) a_{k i} \\
=\frac{\partial}{\partial q_{j}}\left(\sum_{k=0}^{n-1} f_{k}(q) a_{k j}\right)=\frac{\partial^{2} u^{\prime}}{\partial q_{j} \partial \bar{q}_{k}}(q),
\end{array}
$$

where $f_{k}(q):=\left(\overline{\partial u / \partial q_{k}}\right)(A q)=\partial_{\bar{q}_{k}} u(A q)$, and hence (64) follows and the corollary is proved.

\section{Statement of the Theorem}

\subsection{The Main Result of This Paper}

Theorem 13. Let $D \subset \mathbb{R}^{4 n} \cong \mathbb{M}_{s}^{n}$ be a domain and $f: D \rightarrow \mathbb{U}_{s}$. Then

$$
\begin{aligned}
\Delta_{n}(f) & :=(\Delta f)^{n}:=\underbrace{(\Delta f) \wedge(\Delta f) \wedge \cdots \wedge(\Delta f)}_{n \text {-times }} \\
& =n ! \cdot \operatorname{det} \mathscr{H}_{s}(f) \cdot d z_{I},
\end{aligned}
$$

where $I=\{0,1, \ldots, 2 n-1\}$ is a multi-index and $d z_{I}:=d z_{0} \wedge$ $d z_{1} \wedge \cdots \wedge d z_{2 n-1}$ is the holomorphic volume form in $\mathbb{C}^{2 n}$.

3.2. Proof of Theorem 13 for the Case $n=2$. First we prove Theorem 13 for the case $n=2$ (base case) and then proceed by induction.

Proof. Let $n=2$ and consider the embedding $\tau$ from Section 2.1:

$$
\begin{aligned}
\mathbb{M}_{s}^{2} \ni q=\left(\begin{array}{c}
q_{0} \\
q_{1}
\end{array}\right)=\left(\begin{array}{c}
z_{0}+z_{1} \cdot s \\
z_{2}+z_{3} \cdot s
\end{array}\right) \underbrace{\rightleftarrows}_{\tau} \tau(q) \\
=\left(\begin{array}{c}
\tau_{0}\left(q_{0}\right) \\
\tau_{1}\left(q_{1}\right)
\end{array}\right)=\left(\begin{array}{c}
\left(\begin{array}{cc}
\bar{z}_{0} & \bar{z}_{1} \\
z_{1} & z_{0}
\end{array}\right) \\
\left(\begin{array}{ll}
\bar{z}_{2} & \bar{z}_{3} \\
z_{3} & z_{2}
\end{array}\right)
\end{array}\right) .
\end{aligned}
$$

The correspoding operators are

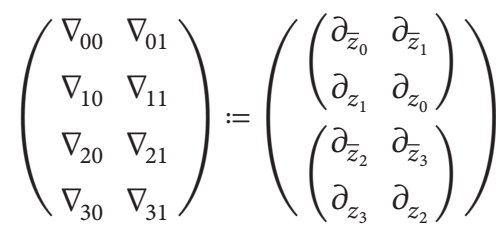

and we use Lemma 3 to complete the proof:

$$
\Delta f=\sum_{0 \leq i<j \leq 3} \Delta_{i j} f d z_{i} \wedge d z_{j}
$$

from which it follows that wedging $\Delta f$ to itself yields

$$
(\Delta f)^{2}:=(\Delta f) \wedge(\Delta f)
$$

where again $I=\{0,1, \ldots, 2(2)-1\}$ and $d z_{I}:=d z_{0} \wedge d z_{1} \wedge$ $d z_{2} \wedge d z_{3}$ is the holomorphic volume form in $\left(\Lambda^{4}\right)^{*} \mathbb{C}^{2(2)}$.

On the other hand (40) tells us how to compute entries in the split-quaternionic Hessian, which works out beautifully:

$$
\begin{aligned}
& \partial_{00}^{q}=\partial_{0 \overline{0}}^{2}-\partial_{\overline{1} 1}^{2}=\Delta_{01}, \\
& \partial_{11}^{q}=\partial_{2 \overline{2}}^{2}-\partial_{\overline{3} 3}^{2}=\Delta_{23}, \\
& \partial_{10}^{q}=\partial_{2 \overline{0}}^{2}-\partial_{\overline{3} 1}^{2}+\left(\partial_{\overline{3} 0}^{2}-\partial_{2 \overline{1}}^{2}\right) \cdot s=-\Delta_{12}+\Delta_{02} \cdot s
\end{aligned}
$$

and when combined with Lemma 3, that gives

$$
\begin{aligned}
& \overline{\Delta_{12}}=-\Delta_{03}, \\
& \overline{\Delta_{02}}=-\Delta_{13} .
\end{aligned}
$$


The third row of the above then also implies that

$$
\begin{aligned}
\left\|\partial_{10}^{q}\right\|^{2} & =\partial_{10}^{q} \cdot \overline{\partial_{10}^{q}}=\left(-\Delta_{12}\right)\left(\overline{-\Delta_{12}}\right)-\left(\Delta_{02}\right)\left(\overline{\Delta_{02}}\right) \\
& =\Delta_{12} \overline{\Delta_{12}}-\Delta_{02} \overline{\Delta_{02}}=\Delta_{02} \Delta_{13}-\Delta_{03} \Delta_{12}
\end{aligned}
$$

so that $(\Delta f)^{2}$ is actually

$$
\begin{aligned}
& (\Delta f)^{2}=2\left[\Delta_{01} f \Delta_{23} f-\left(\Delta_{02} f \Delta_{13} f-\Delta_{03} f \Delta_{12} f\right)\right] \\
& \cdot d z_{I}=2\left[\partial_{00}^{q} f \partial_{11}^{q} f-\partial_{10}^{q} f\left(\overline{\partial_{10}^{q}} f\right)\right] \cdot d z_{I} \\
& =2 \cdot \operatorname{det}\left(\begin{array}{ll}
\partial_{00}^{q} f & \partial_{01}^{q} f \\
\partial_{10}^{q} f & \partial_{11}^{q} f
\end{array}\right) \cdot d z_{I} \\
& =2 \cdot \operatorname{det} \mathscr{H}_{s}(f) \cdot d z_{I}
\end{aligned}
$$

since $\partial_{i j}^{q}=\overline{\partial_{j i}^{q}}$ [see (41)]. This concludes the proof of Theorem 13 for the case $n=2$.

3.3. Proof of Induction. We now assume that Theorem 13 is true for some $n-1 \in \mathbb{N}$; we want to prove by induction that it holds for $n$. We consider a $C^{2}$ function $f\left(q_{0}, \ldots, q_{n-1}\right)$ of $n$ variables that has continuous 2 nd-order mixed partial derivatives. First we prove a result in functional analysis regarding the density of delta functions (on hyperplanes) in the space of (tempered) distributions, implying that the span of said delta functions contains the set of smooth functions, which are dense in the $C^{2}$ functions.

Lemma 14. Linear combinations of delta functions is dense in the space of generalized functions $\mathscr{D}\left(\mathbb{W}_{s}^{n}\right)=\left(\mathcal{S}\left(\mathbb{W}_{s}^{n}\right)\right)^{*}$, where $\mathcal{S}\left(\mathbb{H}_{s}^{n}\right)$ is the Schwartz space of rapidly decreasing functions on $\mathbb{W}_{s}^{n}$.

Proof. Consider the Fréchet space $\mathcal{S}\left(\mathbb{H}_{s}^{n}\right)$ with the Fréchet topology and its dual space $\mathscr{D}\left(\mathbb{U}_{s}^{n}\right)$. We wish to show that the Schwartz space $\mathcal{S}\left(\mathbb{U}_{s}^{n}\right)$ is dense in $\left(\mathscr{D}\left(\mathbb{V}_{s}^{n}\right)\right)^{*}$, the dual of the distribution space. It is well known that the evaluation map is an injection from a topological vector space $X$ into its double dual $X^{* *}$; hence for $X=\mathcal{S}\left(\mathbb{H}_{s}^{n}\right)$ we have a copy of $\mathcal{S}\left(\mathbb{H}_{s}^{n}\right) \subset\left(\mathscr{D}\left(\mathbb{M}_{s}^{n}\right)\right)^{*}=\left(\mathcal{S}\left(\mathbb{T}_{s}^{n}\right)\right)^{* *}$.

But since $\mathcal{S}\left(\mathbb{G}_{s}^{n}\right)$ is a nuclear Fréchet space which is also barreled (see [16], pg. 107, 147) then $\mathcal{S}\left(\mathbb{H}_{s}^{n}\right)$ is (semi)reflexive; that is, $\mathcal{S}\left(\mathbb{U}_{s}^{n}\right) \cong\left(S\left(H_{s}^{n}\right)\right)^{* *}=\left(\mathscr{D}\left(\mathbb{U}_{s}^{n}\right)\right)^{*}$ as vector spaces and hence the Schwartz space is trivially dense in the dual of $\mathscr{D}\left(\mathbb{U}_{s}^{n}\right)$. Consider the subspace

$$
\text { S }
$$

$$
=\operatorname{span}\left\{\delta_{E} \mid E \subset \mathbb{H}_{s}^{n} \text {-split-quaternionic hyperplane }\right\}
$$

and its closure $\bar{S}$ inside $\left(\mathscr{D}\left(\mathbb{W}_{s}^{n}\right)\right)^{*}$, and suppose $\bar{S} \neq\left(\mathscr{D}\left(\mathbb{M}_{s}^{n}\right)\right)^{*}$. By the Hahn-Banach theorem, there is a linear functional $l$ such that $\left.l\right|_{S} \equiv 0$ and $\left.l\right|_{\left(\mathscr{D}\left(\llbracket_{s}^{n}\right)\right)^{*}-\bar{S}}>0$. Thus there exists a nonzero Schwartz function $g$ such that the functional

$$
l: f \longmapsto l(f):=\int_{\mathbb{Q}_{s}^{n}} g \cdot f \neq 0 .
$$

But then for any (all) $\delta_{E} \in S$,

$$
l\left(\delta_{E}\right)=\int_{\mathbb{Q}_{s}^{n}} g \cdot \delta_{E}=\int_{E} g=: \mathscr{R}_{g}(E)=0 \Longleftrightarrow g \equiv 0
$$

since the Radon transform (defined on hyperplanes) $\mathscr{R}_{g}$ : $E \mapsto \int_{E} g$ (for nonzero $g$ ) is injective (proved in $[11,17]$ ), which is a contradiction since $g$ was assumed to be nonzero.

Hence $\bar{S}=\left(\mathscr{D}\left(\mathbb{H}_{s}^{n}\right)\right)^{*}$; that is, the span of delta functions $S$ is dense in $\left(\mathscr{D}\left(\mathbb{T}_{s}^{n}\right)\right)^{*}$.

Proof of Theorem 13 for $n>2$. By Lemma 14 and the properties of the mixed Baston product and mixed discriminant, it suffices to prove

$$
\Delta_{n}\left(f_{1}, \ldots, f_{n}\right)=n ! \cdot \operatorname{det}\left(f_{1}, \ldots, f_{n}\right) d z_{I}
$$

for $n>2$ in the case $f_{1}(q)=\delta_{E}$, where $E=\left\{q \mid \sum_{i} \lambda_{i} q_{i}=0\right\}$ is a split-quaternionic hyperplane, which implies Theorem 13. We proceed by finding a unitary linear transformation $A$ such that $E=\left\{q \mid q_{1}=0\right\}$. We can use the pullback functions $f_{k}^{\prime}\left(q^{\prime}\right)=f_{k}\left(A q^{\prime}\right)$, and by Corollary 12 we have that

$$
\begin{aligned}
F_{k}^{\prime} & :=\left(\frac{\partial^{2} f_{k}^{\prime}}{\partial q_{i}^{\prime} \partial \bar{q}_{j}^{\prime}}\left(q^{\prime}\right)\right)=A\left(\frac{\partial^{2} f_{k}}{\partial q_{i} \partial \bar{q}_{j}}\left(A q^{\prime}\right)\right) A^{*} \\
& =: A \cdot F_{k} \cdot A^{*},
\end{aligned}
$$

where $q:=A q^{\prime}$ and $k=1, \ldots, n$. Then

$$
\begin{aligned}
\sum_{k} x_{k} F_{k}^{\prime} & =\sum_{k} x_{k}\left(A \cdot F_{k} \cdot A^{*}\right) \\
& =A \cdot\left(\sum_{k} x_{k} F_{k}\right) \cdot A^{*} \Longrightarrow \\
\operatorname{det}\left(\sum_{k} x_{k} F_{k}^{\prime}\right) & =\operatorname{det}\left(A \cdot\left(\sum_{k} x_{k} F_{k}\right) \cdot A^{*}\right) \\
& =\operatorname{det}\left(A \cdot A^{*}\right) \cdot \operatorname{det}\left(\sum_{k} x_{k} F_{k}\right) \\
& =\operatorname{det}\left(\sum_{k} x_{k} F_{k}\right)
\end{aligned}
$$

since $A$ is unitary; that is, $A \cdot A^{*}=\mathrm{Id}$. Hence it follows by the definition of the mixed discriminant and using the simpler notation

$$
\begin{aligned}
& \operatorname{det}\left(\delta_{\left\{\sum_{k} \lambda_{k} q_{k}=0\right\}}, f_{2}, \ldots, f_{n}\right)(q) \\
& =\operatorname{det}\left(\delta_{\left\{\sum_{k} \lambda_{k} q_{k}=0\right\}}, f_{2}, \ldots, f_{n}\right)\left(A q^{\prime}\right) \\
& =\operatorname{det}\left(\delta_{\left\{q_{1}^{\prime}=0\right\}}, f_{2}^{\prime}, \ldots, f_{n}^{\prime}\right)\left(q^{\prime}\right),
\end{aligned}
$$

where again $q=A q^{\prime}$. From these considerations, it then suffices to prove (80) in the case where $f_{1}=\delta_{E}$ and $E=$ $\left\{q_{1}=0\right\}$. We can compute the split-quaternionic Hessian 
of $\delta_{E}$, where the derivatives are now weak derivatives of distributions. For any test function $\phi \in C_{0}^{\infty}\left(\mathbb{T}_{s}^{n}\right)$ we have

$$
\begin{aligned}
\frac{\partial \delta_{E}}{\partial \bar{q}_{j}}(\phi) & :=\int_{\mathbb{\mathbb { U } _ { s } ^ { n }}} \phi \cdot \frac{\partial \delta_{E}}{\partial \bar{q}_{j}} d q=-\int_{\mathbb{\mathbb { V } _ { s } ^ { n }}} \frac{\partial \phi}{\partial \bar{q}_{j}} \cdot \delta_{E} \cdot d q \\
& =\left.\int_{E} \frac{\partial \phi}{\partial \bar{q}_{j}} d q\right|_{E},
\end{aligned}
$$

where $d q=\bigwedge_{k=1}^{n} d q_{k}$ is the volume measure on $\mathbb{H}_{s}$ and $\left.d q\right|_{E}=$ $d q_{2} \wedge \cdots \wedge d q_{n}$ is the volume measure on $E$. Translated into the complex representations, $d q=\bigwedge_{k=1}^{2 n} d z_{k} \wedge d \bar{z}_{k}=: d \mathbf{Z}$ and $\left.d q\right|_{E}=\bigwedge_{k=3}^{2 n} d z_{k} \wedge d \bar{z}_{k}=: d \mathbf{Z}_{1}$ so that, for $j=1,2, \ldots, n$,

$$
\begin{aligned}
\left.\frac{\partial \phi}{\partial \bar{q}_{j}} d q\right|_{E} & =\left(\partial_{\overline{2 j-1}} \phi-\partial_{\overline{2 j}} \phi \cdot s\right) d \mathbf{Z}_{1} \\
& =\partial_{\overline{2 j-1}} \phi \cdot d \mathbf{Z}_{1}-\partial_{\overline{2 j}} \phi \cdot d \mathbf{Z}_{1} \cdot s .
\end{aligned}
$$

But letting

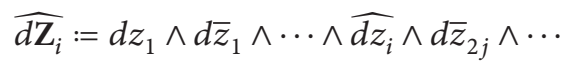

$$
\begin{aligned}
& \wedge d z_{2 n} \wedge d \bar{z}_{2 n} \\
& {\widehat{d \mathbf{Z}_{\bar{i}}}}:=d z_{1} \wedge d \bar{z}_{1} \wedge \cdots \wedge d z_{i} \wedge \widehat{d \bar{z}}_{i} \wedge \cdots \\
& \wedge d z_{2 n} \wedge d \bar{z}_{2 n} \\
& \partial_{\overline{2 j-1}} \phi \cdot d \mathbf{Z}=\sum_{k=1}^{2 n} \delta_{k}^{2 j-1} \frac{\partial \phi}{\partial \bar{z}_{k}} \overline{d \mathbf{Z} \overline{2 j-1}} \wedge d \bar{z}_{k} \\
& =\sum_{k=1}^{2 n} \frac{\partial}{\partial \bar{z}_{k}}\left(\phi \cdot \overline{d \mathbf{Z}_{\overline{2 j-1}}}\right) \wedge d \bar{z}_{k} \\
& +\underbrace{\sum_{k=1}^{2 n} \frac{\partial}{\partial z_{k}}\left(\phi \cdot \widehat{d \mathrm{Z}_{\overline{2 j-1}}}\right) \wedge d z_{k}}_{=0} \\
& =d\left(\phi \cdot \overline{d \overline{\mathbf{Z}_{\overline{2 j-1}}}}\right),
\end{aligned}
$$

which means that $\partial_{\overline{2 j-1}} \phi \cdot d \mathbf{Z}=d\left(\phi \cdot \overline{d \mathbf{Z}_{\overline{2 j-1}}}\right)$ and similarly $\partial_{\overline{2 j}} \phi \cdot d \mathbf{Z}=d\left(\phi \cdot \widehat{d \mathbf{Z}_{\overline{2 j}}}\right)$ are exact forms and hence by Stokes' theorem

$$
\begin{aligned}
\left.\int_{E} \frac{\partial \phi}{\partial \bar{q}_{j}} d q\right|_{E} & =\int_{E}\left(\partial_{\overline{2 j-1}} \phi \cdot d \mathbf{Z}_{1}-\partial_{\overline{2 j}} \phi \cdot d \mathbf{Z}_{1} \cdot s\right) \\
& =\int_{E} \partial_{\overline{2 j-1}} \phi \cdot d \mathbf{Z}_{1}-\int_{E} \partial_{\overline{2 j}} \phi \cdot d \mathbf{Z}_{1} \cdot s \\
& =\int_{E} d\left(\phi \cdot \widehat{d \mathbf{Z}_{\overline{2 j-1}}}\right)-\int_{E} d\left(\phi \cdot \widehat{d \mathbf{Z}_{\overline{2 j}}}\right) \\
& =\int_{\partial E} \phi \cdot \widehat{d \mathbf{Z}_{\overline{2 j-1}}}-\int_{\partial E} \phi \cdot \widehat{d \mathbf{Z}_{\overline{2 j}}} \\
& =\lim _{b \rightarrow \infty}\left(\int_{\partial E_{b}} \phi \cdot \widehat{d \mathbf{Z}_{\overline{2 j-1}}}-\int_{\partial E_{b}} \phi \cdot \widehat{d \mathbf{Z}_{\overline{2 j}}}\right) \\
& =0-0, \quad \text { if } j \neq 1
\end{aligned}
$$

because of the compact support of $\phi$, and $\partial E_{b}=\left\{\left|z_{i}\right| \leq\right.$ $b: i=3,4, \ldots, 2 n\}$. If $j=1$ then since $d \bar{z}_{1}$ and $d \bar{z}_{2}$ do not appear in ${\widehat{d \mathbf{Z}_{\overline{1}}}}_{\mathrm{1}}$ and ${\widehat{d \mathbf{Z}_{\overline{2}}}}_{\overline{2}}$, respectively, the integral and hence $\left(\partial \delta_{E} / \partial \bar{q}_{1}\right)(\phi)$ may not necessarily be zero. Applying the partial derivative with respect to $q_{1}$ to (84) with $j=1$ we get the first entry in the split-quaternionic Hessian matrix for $\delta_{E}$, and combining with (87) we obtain

$$
\begin{aligned}
\left(\frac{\partial^{2} \delta_{E}}{\partial q_{i} \partial \bar{q}_{j}}\right) & =\left(\begin{array}{ccc}
\frac{\partial^{2} \delta_{E}}{\partial q_{1} \partial \bar{q}_{1}} & & 0 \\
& \ddots & \\
0 & & 0
\end{array}\right) \\
& =\left(\begin{array}{ccc}
\Delta_{q_{1}} \delta_{E} & & 0 \\
& \ddots & \\
0 & & 0
\end{array}\right)
\end{aligned}
$$

since $\partial^{2} \delta_{E} / \partial q_{i} \partial \bar{q}_{j}=0$ if $i \neq 1 \neq j$, and the second equality is by definition for $n=1$. Using Corollary 10 we have

$$
\begin{aligned}
& n \cdot \operatorname{det}\left(\delta_{E}, f_{2}, \ldots, f_{n}\right) \\
& \quad=\Delta_{q_{1}} \delta_{E} \cdot \operatorname{det}\left(M_{\{1\}} F_{2}, \ldots, M_{\{1\}} F_{n}\right),
\end{aligned}
$$

where $M_{\{1\}} F_{k}=\left(\partial^{2} f_{k} / \partial q_{i} \partial \bar{q}_{j}\right)_{i, j=2, \ldots, n}$ are the corresponding hyperhermitian minors of the original matrices. Then from (89) it follows that for any test function $\phi \in C_{0}^{\infty}\left(\mathbb{M}_{s}^{n}\right)$ we have

$$
\begin{aligned}
& \int_{\mathbb{\mathbb { U } _ { s } ^ { n }}} \phi \cdot \operatorname{det}\left(\delta_{E}, f_{2}, \ldots, f_{n}\right) \\
& \quad=\int_{\mathbb{U}_{s}^{n}} \frac{1}{n} \cdot \phi \cdot \Delta_{q_{1}} \delta_{E} \cdot \operatorname{det}\left(M_{\{1\}} F_{2}, \ldots, M_{\{1\}} F_{n}\right) \\
& =\left.\int_{E} \frac{1}{n} \cdot \Delta_{q_{1}}\left[\phi \cdot \operatorname{det}\left(M_{\{1\}} F_{2}, \ldots, M_{\{1\}} F_{n}\right)\right]\right|_{q_{1}=0} .
\end{aligned}
$$

Since the domain of integration is $E=\left\{q_{1}=0\right\}$, the integral only depends on no more than 2 nd-order derivatives of $f_{2}, \ldots, f_{n}$ in the direction of $q_{1}$. Thus we can assume that there exists polynomials $p_{k}$ of order no more than 2 such that $f_{k}\left(q_{1}, \ldots, q_{n}\right)=p_{k}\left(q_{1}\right) \widehat{f}_{k}\left(q_{2}, \ldots, q_{n}\right)$ for $k=2,3, \ldots, n$.

Let $M_{\{1\}} \widehat{F}_{k}$ denote $\left(\partial^{2} \widehat{f}_{k} / \partial q_{i} \partial \bar{q}_{j}\right)_{i, j=2, \ldots, n}$, and then it follows that

$$
\begin{aligned}
\operatorname{det} & \left(M_{\{1\}} F_{2}, \ldots, M_{\{1\}} F_{n}\right) \\
= & p_{2}\left(q_{1}\right) \cdots p_{n}\left(q_{1}\right) \cdot \operatorname{det}\left(M_{\{1\}} \widehat{F}_{2}, \ldots, M_{\{1\}} \widehat{F}_{n}\right) \\
= & p_{2}\left(q_{1}\right) \cdots p_{n}\left(q_{1}\right) \cdot \operatorname{det}\left(\widehat{f}_{2}, \ldots, \widehat{f}_{n}\right) .
\end{aligned}
$$


Thus we obtain

$$
\begin{aligned}
\int_{\mathbb{Q}_{s}^{n}} \phi \cdot \operatorname{det}\left(\delta_{E}, f_{2}, \ldots, f_{n}\right) \\
=\left.\int_{E} \frac{1}{n} \cdot \Delta_{q_{1}}\left[\phi \cdot \operatorname{det}\left(M_{\{1\}} F_{2}, \ldots, M_{\{1\}} F_{n}\right)\right]\right|_{q_{1}=0} \\
=\left.\int_{E} \frac{1}{n !} \cdot \Delta_{q_{1}}\left[\phi \cdot p_{2}\left(q_{1}\right) \cdots p_{n}\left(q_{1}\right)\right]\right|_{q_{1}=0} \\
\cdot \Delta_{n-1}\left(\hat{f}_{2}, \ldots, \hat{f}_{n}\right) .
\end{aligned}
$$

On the other hand, using properties of the mixed Baston product and our inductive hypothesis used in the last equality we have

$$
\begin{aligned}
& \int_{\mathbb{U}_{s}^{n}} \phi \cdot \Delta_{n}\left(\delta_{E}, f_{2}, \ldots, f_{n}\right)=\int_{\mathbb{U}_{s}^{n}} \phi \cdot \sum_{i_{1}, j_{1}, \ldots} \delta_{1,2,3,4, \ldots, 2 n}^{i_{1} j_{1} i_{2} j_{2}, \ldots, i_{n} j_{n}} \\
& \cdot \Delta_{i_{1} j_{1}} \delta_{E} \cdots \Delta_{i_{n} j_{n}} f_{n}=\int_{\mathbb{R}_{s}^{n}} 2 \phi \\
& \cdot \sum_{i_{1}, j_{1}, \ldots} \delta_{1,2,3,4, \ldots, 2 n}^{12 i_{2} j_{2}, \ldots, i_{n} j_{n}} \Delta_{12} \delta_{E} \cdots \Delta_{i_{n} j_{n}} f_{n}=\int_{\mathbb{Q} \square_{s}^{n}} \phi \cdot \Delta_{q_{1}} \delta_{E} \\
& \cdot p_{2}\left(q_{1}\right) \cdots p_{n}\left(q_{1}\right) \\
& \cdot \sum_{i_{2}, j_{2}, \ldots} \delta_{1,2,3,4, \ldots, 2 n}^{12 i_{2} j_{2}, \ldots, i_{n} j_{n}} \Delta_{i_{2} j_{2}} \widehat{f}_{2} \cdots \Delta_{i_{n} j_{n}} \hat{f}_{n} \\
& =\left.\int_{\mathbb{\mathbb { U } _ { s } ^ { n }}} \Delta_{q_{1}}\left[\phi \cdot p_{2}\left(q_{1}\right) \cdots p_{n}\left(q_{1}\right)\right]\right|_{q_{1}=0} \\
& \cdot \sum_{i_{2}, j_{2}, \ldots} \delta_{1,2,3,4, \ldots, 2 n}^{12 i_{2} j_{2}, \ldots, i_{n} j_{n}} \Delta_{i_{2} j_{2}} \widehat{f}_{2} \cdots \Delta_{i_{n} j_{n}} \widehat{f}_{n} \\
& =\left.\int_{\mathbb{R}_{s}^{n}} \Delta_{q_{1}}\left[\phi \cdot p_{2}\left(q_{1}\right) \cdots p_{n}\left(q_{1}\right)\right]\right|_{q_{1}=0} \\
& \cdot \Delta_{n-1}\left(\widehat{f}_{2}, \ldots, \widehat{f}_{n}\right)=\int_{E} \frac{1}{n !} \\
& \left.\cdot \Delta_{q_{1}}\left[\phi \cdot p_{2}\left(q_{1}\right) \cdots p_{n}\left(q_{1}\right)\right]\right|_{q_{1}=0} \\
& \cdot \Delta_{n-1}\left(\hat{f}_{2}, \ldots, \hat{f}_{n}\right) \text {. }
\end{aligned}
$$

Hence combining (93) with (92) we get that the integrands are equal almost everywhere, but since the functions are continuous, we have equality, and Theorem 13 is proved.

\section{Split Quaternions and Structures on Manifolds}

The operator $\Delta$ above can be generalized for any manifold with a special structure which we call split-hypercomplex (other known names are parahypercomplex and neutral hypercomplex). Let $M$ be a manifold and let $I$ be a complex structure on it; that is, $I: T M \rightarrow T M, I^{2}=-I d$ is integrable almost complex structure. Suppose also that there is $S: T M \rightarrow T M$ with $S^{2}=I d$ and $I S=-S I$. If the \pm 1 eigen-bundles of $S$ are involutive, $S$ is called integrable. When $S$ is integrable, $T=I S$ again has $T^{2}=I d$ and it is known that it is integrable. We call such $(M, I, S, T)$ with integrable $I, S, T$ split-hypercomplex manifold and $(I, S, T)$ split-hypercomplex structure. Clearly the left multiplication by $i, s, t$ in $\mathbb{T}_{s}^{n}$ provides such a structure. However, unlike the complex manifolds, split-hypercomplex ones do not have nice atlases with "spli-quaternionic-holomorphic" transition functions, so the local considerations of the previous section cannot be extended to an arbitrary manifold. For any function $f: M \rightarrow \mathbb{R}$, however we can define an analog of the Baston operator $\Delta$. Denote by $\partial$ and $\bar{\partial}$ the standard operators for the structure $I$. Then $\Delta f=\partial S \bar{\partial} f$ is a globally defined 2-form on $M$, which is of type $(2,0)$ with respect to $I$.

It is known that when $\partial \circ S \circ \bar{\partial} f$ is nondegenerate it defines a pseudo-Riemannian metric $g$ on $M$ of split signature, such that $I$ is an isometry and $S, T$ are anti-isometries of $g$, called split-hyperhermitian. Any split-hyperhermitian structure defines 3 nondegenerate 2 -forms by $\omega_{I}(X, Y)=$ $g(I X, Y), \omega_{S}(X, Y)=g(S X, Y), \omega_{T}(X, Y)=g(T X, Y)$, for which $\omega_{S}+i \omega_{T}$ is nondegenerate $(2,0)$-form with respect to $I$. In particular such metric is necessary of split signature and $M$ has dimension divisible by four. The relation with a function $f$ as above is $\partial \circ S \circ \bar{\partial} f=\omega_{S}+i \omega_{T}$ and conversely, from nondegenerate form $\partial \circ S \circ \bar{\partial} f$ on a split-hypercomplex manifold, one recovers $g$.

However not every hyperhermitian metric arises in such a way. There is an additional integrability condition on $g$ which is obtained as follows: If $\omega_{S}+i \omega_{T}=\partial S \bar{\partial} f$ for some $f$, then $\partial\left(\omega_{S}+i \omega_{T}\right)=0$. The condition is also equivalent to existence of a connection $\nabla$ on $M$ for which $\nabla g=\nabla I=\nabla S=\nabla T=$ 0 and $g\left(T^{\nabla}(X, Y), Z\right)$ is totally skew-symmetric, where $T^{\nabla}$ is the torsion of $\nabla[10]$. On a split-hyperhermitian manifold $M$ admitting such connection with skew-torsion, such function $f$ locally always exists [10] but may not exist globally.

The main result of Section 4 then gives that on $\mathbb{H}_{s}^{n} \cong \mathbb{C}^{2 n}$

$$
(\partial \circ S \circ \bar{\partial} f)^{n}=\operatorname{det}\left(\mathscr{H}_{s} f\right) d z_{1} \wedge \cdots \wedge d z_{2 n} \text {, }
$$

where $\mathscr{H}_{s}$ is the split-quaternionic Hessian of $f$. In the quaternionic case, this gives rise to the so-called quaternionic Monge-Amperè equation, which arises if we want to find $f$ for which the determinant of the quaternionic Hessian is a given function. The quaternionic Monge-Amperè equation is elliptic. In the split-quaternionic case, however the corresponding equation is ultrahyperbolic and is not well studied. On the other side the reduction of self-duality equations in split signature to two dimensions leads to the equations of [5] describing the deformations of a harmonic map from a Riemann surface into compact Lie group, which are elliptic. In $\mathbb{M}_{s}^{n}$ natural geometric objects to study are also the split special Lagrangian submanifolds as studied in [12]. The description in our terminology is the following. Consider the form $\Omega=\omega_{I}-s \omega_{T}$ which has values in split-complex numbers $\mathbf{D}=\left\{x+s y \mid s^{2}=1\right\}$. Then $\Omega^{n}=\Omega_{1}+$ $s \Omega_{2}$ for real nondegenerate $2 n$-forms $\Omega_{1}$ and $\Omega_{2}$. Moreover, when the structure is hypersymplectic, forms $\Omega_{1}$ and $\Omega_{2}$ 
are closed. A split special Lagrangian manifold (of phase zero) then is defined as a submanifold $M$ of $\mathbb{Q}_{s}^{n}$ of real dimension $2 n$, for which the form $\Omega_{2}$ vanishes on $T M$ and $\Omega_{1}$ is nondegenerate. Such manifold is necessarily complex, since its tangent bundle is preserved by $I$. This is a partial case of split special Lagrangian manifolds, which are analogs of the holomorphic Lagrangian submanifolds in hyperkähler manifold.

\section{Conflict of Interests}

The authors declare that there is no conflict of interests regarding the publication of this paper.

\section{Acknowledgment}

The research of Gueo Grantcharov is partially supported by grant from Simons Foundation (no. 246184).

\section{References}

[1] R. S. Ward and M. Tabor, "Integrable and solvable systems, and relations among them [and discussion]," Philosophical Transactions of the Royal Society A: Mathematical, Physical and Engineering Sciences, vol. 315, no. 1533, pp. 451-457, 1985.

[2] H. Ooguri and C. Vafa, "Geometry of $N=2$ strings," Nuclear Physics B, vol. 361, no. 2, pp. 469-518, 1991.

[3] H. Ooguri and C. Vafa, "Self-duality and $N=2$ string magic," Modern Physics Letters A, vol. 5, pp. 1389-1398, 1990.

[4] S. Alesker, "Non-commutative linear algebra and plurisubharmonic functions of quaternionic variables," Bulletin des Sciences Mathématiques, vol. 127, no. 1, pp. 1-35, 2003.

[5] N. Hitchin, "Hypersymplectic quotiens," Atti della Accademia delle Scienze di Torino. Classe di Scienze Fisiche, Matematiche e Naturali, vol. 124, pp. 169-180, 1990.

[6] H. Kamada, "Neutral hyperkähler structures on primary Kodaira surfaces," Tsukuba Journal of Mathematics, vol. 23, no. 2, pp. 321-332, 1999.

[7] N. Blažić and S. Vukmirović, "Para-hypercomplex structures on a four-dimensional Lie group," in Contemporary Geometry and Related Topics, pp. 41-56, World Scientific Publishing, River Edge, NJ, USA, 2004.

[8] J. Davidov, G. Grantcharov, O. Mushkarov, and M. Yotov, "Parahyperhermitian surfaces," Bulletin Mathématiques de la Société des Sciences Mathématiques de Roumanie, vol. 52, no. 100, pp. 281-289, 2009.

[9] M. Dunajski and M. West, "Anti-self-dual conformal structures in neutral signature," in Recent Developments in PseudoRiemannian Geometry, D. V. Alekseevsky and H. Baum, Eds., ESI Lectures in Mathematics and Physics, pp. 113-148, European Mathematical Society, Zürich, Switzerland, 2008.

[10] S. Ivanov, V. Tsanov, and S. Zamkovoy, "Hyper-parahermitian manifolds with torsion," Journal of Geometry and Physics, vol. 56, no. 4, pp. 670-690, 2006.

[11] M. I. Graev, "Integral geometry on the space $L^{n}$ where $L$ is a Matrix Ring," Functional Analysis and Its Applications, vol. 30, no. 4, pp. 277-280, 1996.

[12] F. Reese Harvey and H. Blaine Lawson Jr., "Split special Lagrangian geometry," in Metric and Differential Geometry, vol.
297 of Progress in Mathematics, pp. 43-89, Springer, Basel, Switzerland, 2012.

[13] D. Wan and W. Wang, "On quaternionic Monge-Ampère operator, closed positive currents and Lelong-Jensen type formula on quaternionic space," http://arxiv.org/abs/1401.5291v1.

[14] H. Aslaksen, "Quaternionic determinants," The Mathematical Intelligencer, vol. 18, no. 3, pp. 57-65, 1996.

[15] F. Dyson, "Quaternionic determinants," Helvetica Physica Acta, vol. 45, pp. 289-302, 1972.

[16] H. H. Schaefer, Topological Vector Spaces, Springer, Berlin, Germany, 1971.

[17] I. M. Gelfand, M. I. Graev, and N. Ya. Vilenkin, Generalized Functions-Vol 5: Integral Geometry and Representation Theory, Translated by: E. Saletan, Academic Press, New York, NY, USA, 1966. 


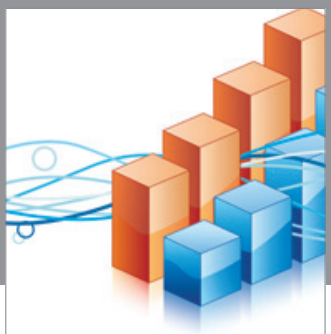

Advances in

Operations Research

vatem alat4

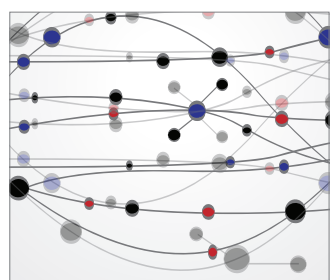

\section{The Scientific} World Journal
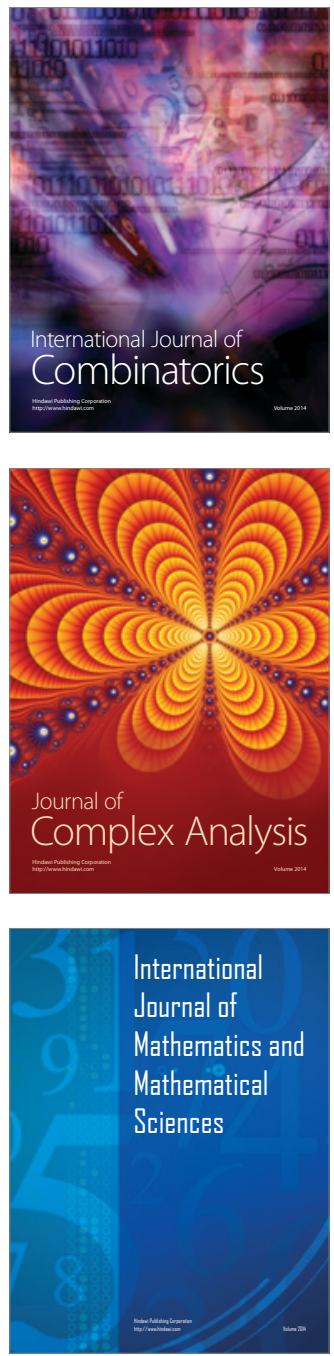
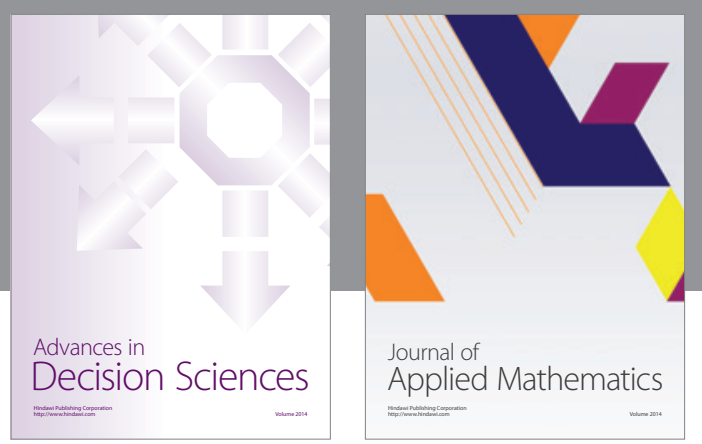

Algebra

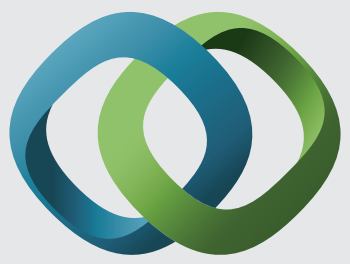

\section{Hindawi}

Submit your manuscripts at

http://www.hindawi.com
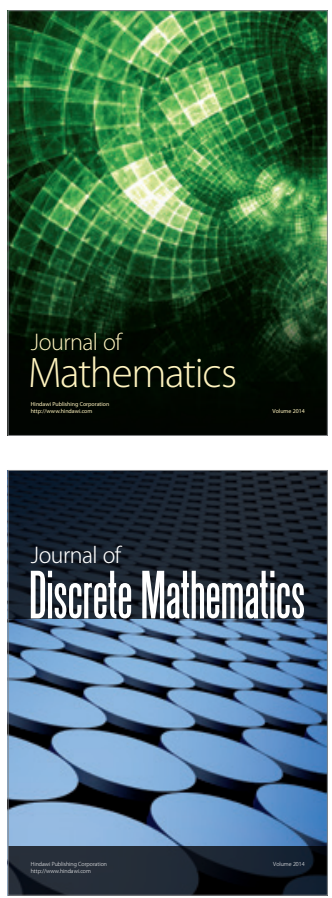

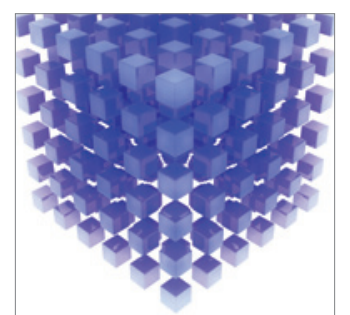

Mathematical Problems in Engineering
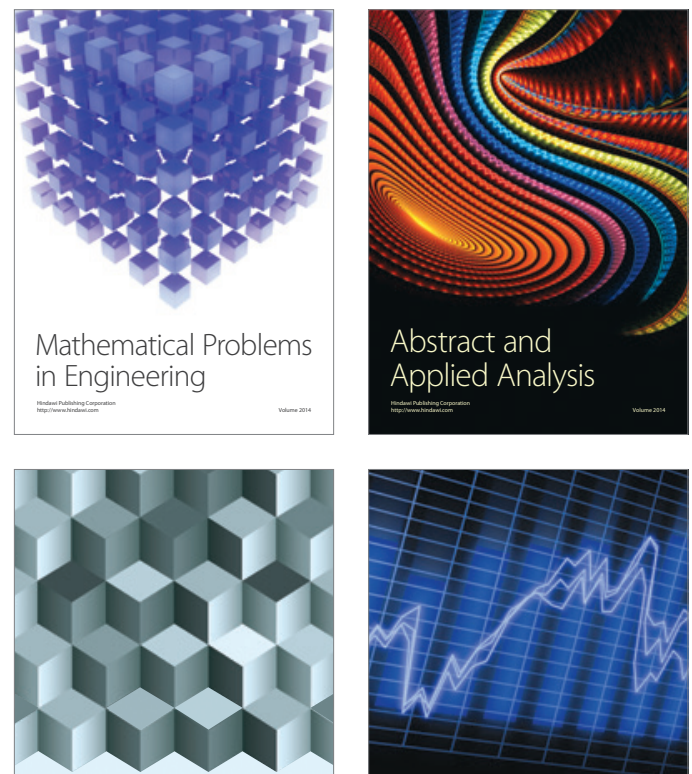

Journal of

Function Spaces

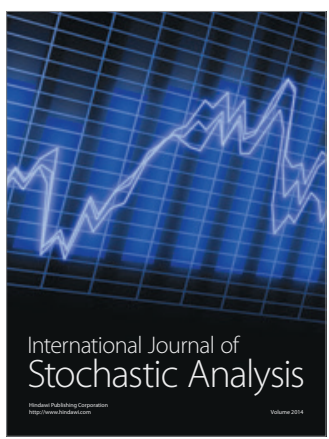

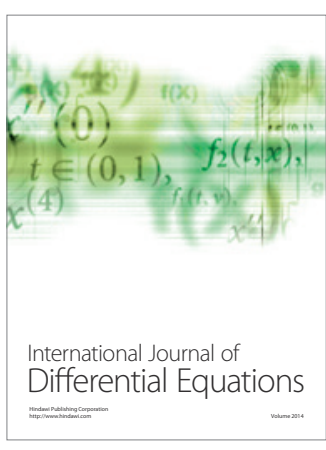
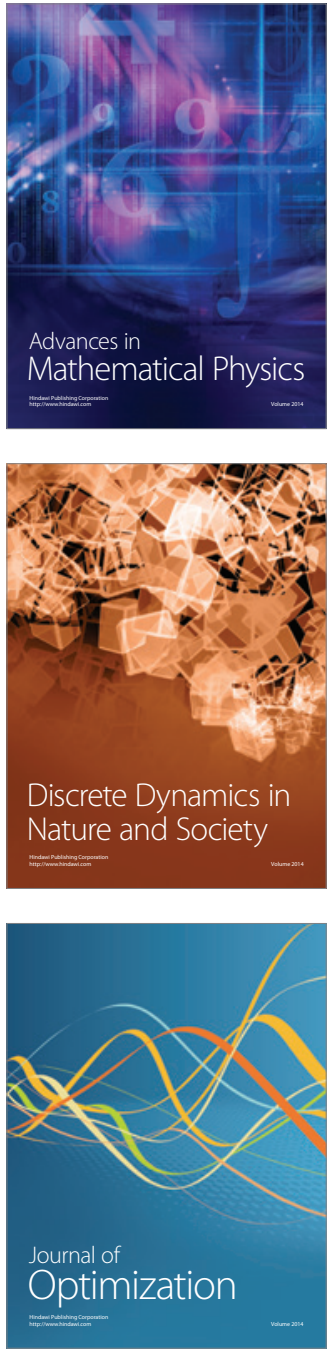